\title{
Domination in Geometric Intersection Graphs
}

\author{
Thomas Erlebach $^{1}$ and Erik Jan van Leeuwen ${ }^{2, \star}$ \\ ${ }^{1}$ Department of Computer Science, University of Leicester, \\ University Road, Leicester LE1 7RH, UK, T. Erlebach@mcs. le.ac.uk \\ ${ }^{2}$ CWI, Kruislaan 413, 1098 SJ Amsterdam, the Netherlands, E. J.van. Leeuwen@ Cwi .n I
}

\begin{abstract}
For intersection graphs of disks and other fat objects, polynomial-time approximation schemes are known for the independent set and vertex cover problems, but the existing techniques were not able to deal with the dominating set problem except in the special case of unit-size objects. We present approximation algorithms and inapproximability results that shed new light on the approximability of the dominating set problem in geometric intersection graphs. On the one hand, we show that for intersection graphs of arbitrary fat objects, the dominating set problem is as hard to approximate as for general graphs. For intersection graphs of arbitrary rectangles, we prove APX-hardness. On the other hand, we present a new general technique for deriving approximation algorithms for various geometric intersection graphs, yielding constant-factor approximation algorithms for $r$-regular polygons, where $r$ is an arbitrary constant, for pairwise homothetic triangles, and for rectangles with bounded aspect ratio. For arbitrary fat objects with bounded ply, we get a $(3+\epsilon)$-approximation algorithm.
\end{abstract}

\section{Introduction}

We study the approximability of the minimum dominating set problem in geometric intersection graphs. Given an undirected graph $G=(V, E)$, a set $D \subseteq V$ is a dominating set if every $v \in V$ is in $D$ or has a neighbor in $D$. The aim of Minimum Dominating Set (MDS) is to compute for a given graph a dominating set of minimum cardinality. Although for general graphs the approximability of MDS has been settled [15, 8], the problem is open for numerous graph classes, such as geometric intersection graphs.

Geometric intersection graphs are graphs in which the vertices represent geometric objects and two vertices are adjacent if the corresponding objects intersect. Studying approximation algorithms for fundamental graph optimization problems on such graphs has led to several new techniques, in particular the geometric shifting technique [17], which can be used to obtain polynomial-time approximation schemes (PTASs) for a number of problems, such as Maximum Independent Set and Minimum Vertex Cover in unit disk graphs [18] and in general disk graphs $[13,6,26]$. These algorithms extend to any constant number of dimensions and arbitrary fat objects (including e.g. squares or other regular polygons in the two-dimensional case).

Interestingly, as pointed out in [13], these techniques do not seem sufficient for handling MDS in intersection graphs of objects of different sizes. To the best of our knowledge, there are no results for intersection graphs of disks, squares, etc. beyond

\footnotetext{
* Partially supported by the Dutch BSIK/BRICKS project.
} 
the $(1+\ln n)$-approximation ratio that can be achieved by the greedy algorithm. In particular, we know of no constant-factor approximation algorithm or approximation hardness results. In this paper, we address this open problem by studying the minimum dominating set problem for intersection graphs of different types of fat objects and providing new insights into its approximability.

In Sect. 3 we present a new general approach to deriving approximation algorithms for MDS on geometric intersection graphs. We apply it to obtain the first constantfactor approximation algorithms for MDS on intersection graphs of $r$-regular polygons, of pairwise homothetic triangles, and of rectangles of bounded aspect-ratio.

We also obtain a constant-factor approximation algorithm for MDS on disk graphs of constant ply (see Sect. 4). A surprising corollary of this is a constant integrality gap for MDS on planar graphs. For disk graphs of bounded ply, this result can be improved to a $(3+\epsilon)$-approximation algorithm by using a new variant of the shifting technique. This algorithm extends to intersection graphs of arbitrary fat objects of bounded ply.

The type of fat objects considered impacts the approximability of MDS: We prove that for $n$ arbitrary fat objects, approximation ratio $(1-\epsilon) \ln n$ is not achievable for any $\epsilon>0$, unless $N P \subset D T I M E\left(n^{O(\log \log n)}\right)$. We also solve an open problem of Chlebík and Chlebíková [9], who asked whether their APX-hardness results for intersection graphs of $d$-dimensional axis-parallel boxes extend to the case $d=2$. We affirm this by showing that MDS is APX-hard for rectangle intersection graphs.

\subsection{Known Results}

MDS in general graphs is essentially equivalent to Minimum Set Cover. For $n$-vertex graphs, approximation ratio $1+\ln n$ is achievable by a greedy algorithm, and one cannot get ratio $(1-\epsilon) \ln n$ for any $\epsilon>0$, unless $N P \subset D T I M E\left(n^{O(\log \log n)}\right)[15,8]$.

Even though geometric intersection graphs have properties exploitable to approximate several problems $[13,6,26]$, only few approximation algorithm are known for MDS in such graphs. For unit disk graphs, Marathe et al. [22] gave a constant-factor approximation algorithm, before a PTAS was presented by Hunt et al. [18] and Nieberg et al. [25]. MDS in unit disk graphs seems harder in the weighted than in the unweighted case, but has a constant-factor approximation algorithm by Ambühl et al. [2].

On the negative side, MDS cannot have an FPTAS (unless $\mathrm{P}=\mathrm{NP}$ ), as it is NP-hard for geometric intersection graphs (even for simple classes such as unit disk graphs [10]). Chlebík and Chlebíková [9] have shown that for any $d \geq 3$, Minimum Dominating Set and several other problems are APX-hard on intersection graphs of $d$-dimensional axisparallel boxes. It follows from Marx [23] that Minimum Dominating Set cannot have an EPTAS (Efficient PTAS) for unit square/disk graphs (unless FPT=W[1]).

Some of our algorithms use $\epsilon$-nets, which were used to approximate geometric optimization problems before, e.g. geometric hitting set [5,14], geometric set cover [11].

\section{Preliminaries}

A $\rho$-approximation algorithm for a minimization problem is an algorithm that runs in polynomial time and always produces a solution whose value is at most $\rho \cdot O P T$, 
where $O P T$ is the optimal objective value. The value $\rho$ is also referred to as the approximation ratio. An algorithm that achieves approximation ratio $1+\epsilon$, for arbitrary $\epsilon>0$, and whose running-time is polynomial in the size of the input for any fixed $\epsilon$, is called a polynomial-time approximation scheme or PTAS. If its running-time is polynomial also in $\frac{1}{\epsilon}$, it is called a fully polynomial-time approximation scheme or FPTAS. A c-asymptotic fully polynomial-time approximation algorithm or $c$-FPTA $A^{\omega}$ is an algorithm giving for any $\epsilon>0$ a feasible solution in time polynomial in $\frac{1}{\epsilon}$ and the size of the input, such that the objective value of the solution is at most $(c+\epsilon) O P T$ if the size of the input is at least $c_{\epsilon}$, where $c_{\epsilon}$ is a constant depending only on $\epsilon$. If $c=1$, it is called an asymptotic fully polynomial-time approximation scheme or FPTAS ${ }^{\omega}$.

\section{$2.1 \epsilon$-Nets}

Our main algorithmic results rely on the availability of small $\epsilon$-nets. Given a universe $\mathbb{U}$ and a family $\mathcal{S}$ of $n$ subsets of $\mathbb{U}$ (called objects), we say $\mathcal{R} \subseteq \mathcal{S}$ is an $\epsilon$-net for $\mathcal{S}$ if any element $u \in \mathbb{U}$ covered by more than $\epsilon|\mathcal{S}|$ sets in $\mathcal{S}$ is also covered by $\mathcal{R}$ (i.e., covered by $\bigcup \mathcal{R}$ ). The size of the net is equal to the cardinality of $\mathcal{R}$. Suppose that for objects of a certain type (e.g. disks in the plane), we have a decomposition bound function $f(n)$ bounding the number of simple regions in a canonical decomposition of the complement of the union of $n$ such objects. Then Clarkson and Varadarajan have proved the following result.

Theorem 1 ([11]). For any $0<\epsilon \leq 1$, there is an $\epsilon$-net for $\mathcal{S}$ of size $O(f(1 / \epsilon)+1 / \epsilon)$.

Such a net can be found by a randomized algorithm with polynomial expected running time. For details and a formal definition of $f$, we refer to [11]. By derandomizing the algorithm using the method of conditional expectations, we obtain the following result.

Theorem 2. For any $0<\epsilon \leq 1$, we can find an $\epsilon$-net for $\mathcal{S}$ of size $O(f(1 / \epsilon)+1 / \epsilon)$ in time polynomial in $|\mathcal{S}|, 1 / \epsilon$, and $f(1 / \epsilon)$.

Pseudo-disks (subsets of the plane bounded by simple closed Jordan curves where each pair of curves intersects at most twice) have a linear decomposition bound function [19, 11], giving a linear sized net.

\section{Domination in Geometric Intersection Graphs}

We introduce the novel notion of $\preceq$-dominating sets, which we use with $\epsilon$-nets to approximate geometric dominating set. Let $\preceq$ be a binary reflexive relation on the vertices of a graph $G=(V, E)$. An example, say for geometric intersection graphs, is that $u \preceq v$ if the object representing $u$ is at most as large as the object representing $v$. We call $v \in V$ $\preceq$-larger than $u \in V$ if $u \preceq v$. Denote by $N_{\preceq}(u)=\{v \in V \mid(u, v) \in E, u \preceq v\}$ the set of $\preceq$-larger neighbors of $u$ and $u$ 's closed $\preceq$-larger neighborhood by $N_{\preceq}[u]=$ $N_{\preceq}(u) \cup\{u\}$.

Call a set $D \subseteq V$ a $\preceq$-dominating set if for any $u \in V, u \in D$ or there is a $\preceq$-larger neighbor of $u$ in $D$, i.e. $D \cap N_{\preceq}[u] \neq \emptyset$. In light of the following theorem, we will be interested in binary reflexive relations $\preceq$ where the $\preceq$-factor (the size of a minimum $\preceq$ dominating set divided by the size of a minimum dominating set) is at most a constant. 
Theorem 3 (Main Theorem). Let $G$ be the intersection graph of a set $\mathcal{S}=\left\{s_{u} \subseteq\right.$ $\left.\mathbb{R}^{d} \mid u \in V(G)\right\}$ of closed topological balls with decomposition bound function $f$. Let $\preceq$ be a binary reflexive relation on the vertices of $G$ with $\preceq$-factor $c_{1}$ such that for any vertex $u$ there exist $c_{2}$ points in $s_{u}$ jointly hitting all objects $s_{v}$ with $v \in N_{\prec}(u)$. If the size of a minimum dominating set of $G$ is $k$, then we can find in polynomial time a dominating set of size $O\left(f\left(2 c_{1} c_{2} k\right)+c_{1} c_{2} k\right)$.

Proof. Solve the LP-relaxation of the $\preceq$-dominating set problem. The integer LP is

$$
\begin{array}{cll}
z_{I}^{*}=\min & \sum_{u \in V} x_{u} & \\
\text { s.t. } & \sum_{v \in N_{\preceq}[u]} x_{v} \geq 1 \quad & (\forall u \in V) \\
& x_{u} \in\{0,1\} & (\forall u \in V) .
\end{array}
$$

Observe that $z_{I}^{*} \leq c_{1} k$. In the relaxation, the last constraint is replaced by $x_{u} \geq 0(\forall u \in$ $V)$. Let $x^{*}$ be a vector attaining the optimum fractional value $z^{*}$. Since for any vertex $u$ all objects $s_{v}$ with $v \in N_{\preceq}(u)$ can be hit by $c_{2}$ points in $s_{u}$, each $s_{u}$ contains a point $p$ such that $\sum_{v: p \in s_{v}} x_{v}^{*} \geq \overline{1 /} c_{2}$.

Now construct a set $\mathcal{S}^{\prime}$ from $\mathcal{S}$ by taking $\left\lceil x_{u}^{*} \cdot|\mathcal{S}| / z^{*}\right\rceil$ copies of each object $s_{u}$. Following the previous observation, this means that for any object $s \in \mathcal{S}$ there is a point $p$ in $s$ such that at least $|\mathcal{S}| /\left(c_{2} z^{*}\right)$ objects of $\mathcal{S}^{\prime}$ contain $p$. Furthermore,

$\left|\mathcal{S}^{\prime}\right|=\sum_{u \in V}\left\lceil x_{u}^{*} \cdot|\mathcal{S}| / z^{*}\right\rceil<\sum_{u \in V}\left(1+x_{u}^{*} \cdot|\mathcal{S}| / z^{*}\right)=|\mathcal{S}|+\frac{|\mathcal{S}|}{z^{*}} \sum_{u \in V} x_{u}^{*}=2|\mathcal{S}|$.

Applying Theorem 2, we find a set $\mathcal{R}^{\prime} \subseteq \mathcal{S}^{\prime}$ of size $O\left(f\left(2 c_{2} z^{*}\right)+2 c_{2} z^{*}\right)$ such that any point covered by more than $\left|\mathcal{S}^{\prime}\right| /\left(2 c_{2} z^{*}\right)$ objects of $\mathcal{S}^{\prime}$, and thus also any point covered by at least $|\mathcal{S}| /\left(c_{2} z^{*}\right)$ objects of $\mathcal{S}^{\prime}$, is covered by $\mathcal{R}^{\prime}$. Then $\mathcal{R}^{\prime}$ intersects each object of $\mathcal{S}$ and thus $\mathcal{R}^{\prime}$ is a dominating set of $G$. It has size

$$
O\left(f\left(2 c_{2} z^{*}\right)+2 c_{2} z^{*}\right) \leq O\left(f\left(2 c_{2} z_{I}^{*}\right)+c_{2} z_{I}^{*}\right) \leq O\left(f\left(2 c_{1} c_{2} k\right)+c_{1} c_{2} k\right) .
$$

Following Theorem 2, $\mathcal{R}^{\prime}$ can be found in polynomial time.

Hence the integrality gap ${ }^{3}$ of the LP relaxation of $\preceq$-MDS is $O\left(c_{1} f\left(2 c_{2} z^{*}\right) / z^{*}+c_{1} c_{2}\right)$.

In the remainder, we do not distinguish between a vertex $v \in V$ and the geometric object $s_{v}$ it represents, i.e., $v$ can refer both to the vertex and to the geometric object.

Before we can apply Theorem 3, we need more concrete relations $\preceq$. Consider the intersection graph of a set $\mathcal{S}$ of closed topological balls in $\mathbb{R}^{d}$. Define a relation $\preceq$ Leb such that $u \preceq$ Leb $v$ if and only if the Lebesgue measure of $u$ is at most the Lebesgue measure of $v$. Clearly, $\preceq_{\text {Leb }}$ is a (total) preorder (i.e. $\preceq_{\text {Leb }}$ is reflexive and transitive). The following two easy lemmas are sufficient to show that the $\preceq$ Leb -factor is a constant for many intersection graph classes. We use $N(u)$ to denote the set $\{v \mid(u, v) \in E\}$.

Lemma 1. Let $\preceq$ be a binary reflexive relation on the vertices of $G$ such that for any vertex $u$ a minimum $\preceq$-dominating set for $U_{u}=\{v \mid v \npreceq u, v \in N(u)\}$ has size at most $c$. Then the $\preceq$-factor is at most $c+1$.

The observation here is that if $D$ is a dominating set of $G$ and $D_{u}$ is a minimum $\preceq$ dominating set for $U_{u}$, then $D \cup \bigcup_{u \in D} D_{u}$ is a $\preceq$-dominating set. In fact, a bound on the size of a minimum $\preceq$-dominating set for $U_{u}$ is only needed for vertices $u$ appearing in a particular minimum dominating set. 
Lemma 2. Let $\preceq$ be a total preorder on the vertices of $G$ s.t. for any vertex $u$ the size of any independent set of $N_{\preceq}(u)$ is bounded by $c$. Then the $\preceq$-factor is at most $c+1$.

These lemmas also hold for the fractional $\preceq$-factor (the ratio of the value of the optimum fractional $\preceq$-dominating set and fractional dominating set). By Thm. 3, the integrality gap ${ }^{3}$ of MDS is $O\left(f\left(2 c_{2} c_{3} z^{*}\right) / z^{*}+c_{2} c_{3}\right)$ if the fractional $\preceq$-factor is at most $c_{3}$.

\subsection{Regular Polygons}

We apply Theorem 3 to give constant-factor approximation algorithms for Minimum Dominating Set on intersection graphs of regular polygons. We assume the polygons are pairwise homothetic: one polygon can be obtained from another by scaling and translating (i.e. rotations are not allowed). Applying results of Kim, Kostochka, and Nakprasit [20] (bounding sizes of independent sets in neighborhoods of larger objects) and Lemma 2 , we can show that the $\preceq$ Leb -factor is at most 5 for intersection graphs of homothetic parallelograms and at most 6 for intersection graphs of homothetic copies of any other planar convex object (including disks and regular polygons).

For even regular polygons (i.e. $2 r$-regular polygons), $2 r$ points suffice to hit all $\preceq$ Leb-larger neighbors of a vertex (take the corners ${ }^{4}$ of the polygon). As pairwise homothetic regular polygons are pseudo-disks, we can apply Theorem 3 with a linear decomposition bound to yield the following.

Theorem 4. Let $r>0$ be an integer. There is a polynomial-time $O(r)$-approximation algorithm for Minimum Dominating Set on intersection graphs of pairwise homothetic $2 r$-regular polygons.

Corollary 1. Minimum Dominating Set on square intersection graphs is in APX.

Although Theorem 4 also works for intersection graphs of 2-regular polygons (i.e. interval graphs), a linear-time exact algorithm exists in this case [7].

The $\preceq$ Leb relation does not seem sufficient to give a constant-factor approximation algorithm for odd regular polygons, as it is not possible to hit all $\preceq_{\text {Leb }}$-larger neighbors of a vertex by a constant number of points inside the object, even though a constant number of points outside the object would suffice. The algorithm of Theorem 3 does not seem to extend to this case. However, we can introduce a more restrictive relation $\preceq$ such that $u \preceq v$ if in addition to $u \preceq$ Leb $v, v$ also covers a constant fraction of the boundary of $u$ or covers a corner of $u$. For this relation, a constant number of points inside an object suffices to hit all $\preceq$-larger neighbors. We can also show it has a constant $\preceq$-factor for odd regular polygons. Detailed analysis reveals the restriction that $u \preceq$ Leb $v$ is not necessary. So for two vertices $u, v \in V$, define $u \preceq_{1 / 3} v$ if and only if $v$ contains a corner of $u$ or $v$ covers at least one third of a side of $u$. We first consider triangles.

Theorem 5. There is a polynomial-time $O(1)$-approximation algorithm for Minimum Dominating Set on intersection graphs of pairwise homothetic equilateral triangles.

\footnotetext{
${ }^{3}$ The integrality gap is the ratio of the optimum integral and optimum fractional value of an LP.

${ }^{4}$ To disambiguate between vertices of a graph and vertices of a polygon, vertices of a polygon will be referred to as corners.
} 


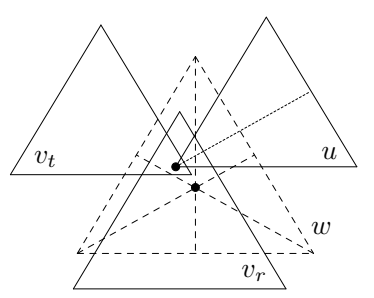

Fig. 1. Triangles $u, v_{t}, v_{r}$, and $w$ of the proof of Theorem 5 . The two dots represent $p$ and the barycenter of $w$.

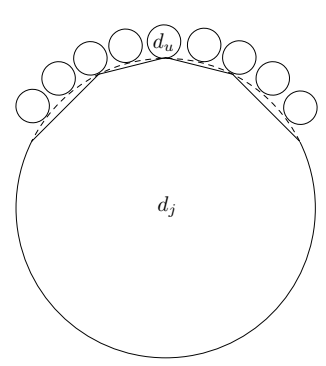

Fig. 2. A cut-off disk $d_{j}$ and the disks $d_{u}$ for elements $u \in \mathbb{U}$ of Theorem 13.

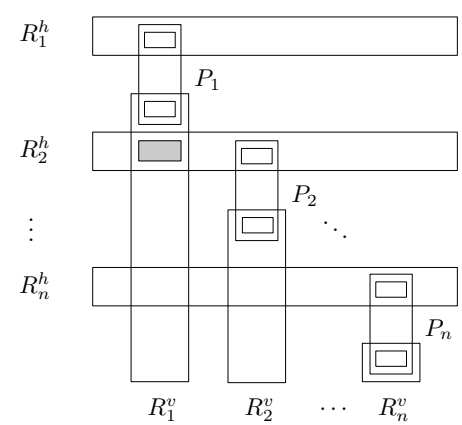

Fig. 3. The intersection graph of Theorem 15. If $\left(v_{1}, v_{2}\right) \in E$, then the shaded rectangle $S_{1,2}$ is in $G^{\prime}$.

Proof. All $\preceq_{1 / 3}$-larger neighbors of a vertex can be hit by 9 points inside the triangle (its three corners and two points equidistantly on each side). To apply the $\preceq_{1 / 3}$ relation with Theorem 3 , we show that the $\preceq_{1 / 3}$-factor is at most 7 . Let $\mathcal{S}$ be a set of equilateral triangles with base parallel to the $x$-axis. Consider a triangle $u$ that is not fully contained in any other triangle of $\mathcal{S}$ and let $U=\left\{v \mid v \npreceq_{1 / 3} u, v \in N(u)\right\}$. For any $v \in U, u$ does not contain a corner of $v$. Hence all $v \in U$ must contain a corner of $u$. Look at one particular corner of $u$, say the left corner, and let $U_{l} \subseteq U$ be the set of triangles intersecting it. Now let $v_{t}$ be a vertex in $U_{l}$ such that the top corner of $v_{t}$ has the largest distance to the altitude ${ }^{5}$ of the left corner of $u$. Similarly, let $v_{r} \in U_{l}$ be a vertex such that the right corner of $v_{r}$ has the largest distance to this altitude (see Fig. 1). We claim $v_{t}, v_{r}$, and $u$ form a $\preceq_{1 / 3}$-dominating set for $U_{l}$.

Let $w$ be a vertex in $U_{l}$. We may assume $w$ has no corner in $v_{t}, v_{r}$, or $u$. Then $w$ contains a corner of $v_{t}, v_{r}$, and $u$. Furthermore, by the choice of $v_{t}$ and $v_{r}, w$ cannot fully contain either $v_{t}$ or $v_{r}$, as the top (right) corner of $w$ would be further from the altitude than the top (right) corner of $v_{t}\left(v_{r}\right)$. Triangles $v_{t}, v_{r}$, and $u$ share a common point $p$ inside $w$ (the leftmost corner of $u$ ). There must be a side of $w$ such that $p$ is at least as far from this side as the barycenter ${ }^{6}$ of $w$. Suppose w.l.o.g. that $v_{r}$ protrudes this side. Then the corner of $v_{r}$ in $w$ is at least as far from this side as $p$, and thus at least as far from the side as the barycenter of $w$. An easy calculation shows that $v_{r}$ covers at least one third of the side of $w$.

Similarly, two triangles can be chosen for the other two corners of $u$. This gives a $\preceq_{1 / 3}$-dominating set for $U$ of size at most 7 . There is a minimum dominating set $D$ such that no triangle in $D$ is strictly contained inside another triangle of $\mathcal{S}$. As we can find a $\preceq_{1 / 3}$-dominating set for $U$ of size at most 7 for any $u$ not fully contained in some other triangle of $\mathcal{S}$, it follows similar to Lemma 1 that the $\preceq_{1 / 3}$-factor is at most 7 .

\footnotetext{
${ }^{5}$ An altitude of a triangle $\tau$ is the line through a corner of $\tau$, perpendicular to the side opposite the corner.

${ }^{6}$ The barycenter or centroid of a triangle is the intersection point of the three straight lines going through a corner of the triangle and the midpoint of the opposite side.
} 
For odd regular polygons with a larger number of sides, a similar proof as in Theorem 5 bounds the $\preceq_{1 / 3}$-factor. However, we can do better. Define a relation $\preceq_{1 / 2}$ such that $u \preceq_{1 / 2} v$ if and only if $v$ contains a corner of $u$ or $v$ covers at least half of a side of $u$. Using the $\preceq_{1 / 2}$ relation yields the following result.

Theorem 6. For any $r \in \mathbb{Z}_{>1}$, there is a polynomial-time $O\left(r^{2}\right)$-approximation algorithm for MDS on intersection graphs of pairwise homothetic $(2 r+1)$-regular polygons.

Our results imply $O(1)$-approximation algorithms for Minimum Connected/Total Dominating Set on intersection graphs of $r$-regular polygons, for constant $r$. Also the results imply a constant bound on the integrality gap of the LP relaxation for these problems, as the bounds on the integral $\preceq_{\text {Leb }^{-},} \preceq_{1 / 3}{ }^{-}$, and $\preceq_{1 / 2}$-factors extend easily to their fractional variants by the fractional versions of Lemma 1 and 2.

\subsection{More General Objects}

The proof of Theorem 5 also goes through for pairwise homothetic triangles in general. Alternatively, one can use an affine transformation to map pairwise homothetic triangles into an equivalent set of pairwise homothetic equilateral triangles (i.e. two mapped triangles intersect if and only if they do so in the original set) and then apply Theorem 5.

Theorem 7. There is a polynomial-time $O(1)$-approximation algorithm for Minimum Dominating Set on intersection graphs of pairwise homothetic triangles.

We also consider intersection graphs of axis-parallel rectangles whose aspect-ratio (the ratio of the length of the longer side over that of the shorter side) is bounded by an integer constant $c$. It is easy to see that any rectangle with aspect-ratio at most $c$ can be represented as the union of at most $c$ squares. Hence the union of $n$ axis-parallel rectangles of aspect-ratio at most $c$ is also the union of $c n$ axis-parallel squares. This implies that the decomposition bound function is $O(\mathrm{cn})$ (as squares are pseudo-disks). Furthermore, $\mathrm{a} \preceq$ Leb -larger rectangle intersecting a rectangle $u$ must contain a corner of $u$ or a $\frac{1}{c}$-fraction of a side of $u$. Hence $O(c)$ points in $u$ suffice to hit all $\preceq$ Leb-larger rectangles intersecting $u$ and the $\preceq$ Leb-factor is $O(c)$. Now apply Theorem 3 .

Theorem 8. For any $c>1$, there is a polynomial-time $O\left(c^{3}\right)$-approximation algorithm for MDS on intersection graphs of axis-parallel rectangles with aspect-ratio at most $c$.

These methods do not seem to extend to intersection graphs in higher dimensions.

\section{Disk Graphs of Bounded Ply}

We do not know how to use the above approach to obtain a constant-factor approximation algorithm for Minimum Dominating Set in general disk graphs, even though the $\preceq$ Leb -factor is 6 . However, this low $\preceq$ Leb -factor can be used to give an approximation algorithm if the ply of the set of disks is bounded. The ply of a set of objects is the maximum over all points $p$ of the number of objects strictly containing $p$ [24].

Using Theorem 3, we can give an $O(\gamma)$-approximation algorithm for MDS on disk intersection graphs of ply $\gamma$. Different techniques however can improve the hidden constant of this result and make it explicit. 
Theorem 9. The integrality gap of the LP relaxation of Minimum Dominating Set on disk intersection graphs of ply $\gamma$ is at most $54 \cdot \gamma$. If the ply is 1 , the gap is at most 42. Hence the gap of the LP relaxation of MDS on planar graphs is at most 42.

Proof (Sketch). We transform the minimum $\preceq$ Leb-dominating set problem on the input graph to a Minimum Set Cover (MSC) instance in which the element frequency is at $\operatorname{most}_{\max _{u \in V}}\left|N_{\preceq \text { Leb }}[u]\right|$. Following a result of Hochbaum [16], the integrality gap of the MSC instance is at most the element frequency. But then the integrality gap of the original problem is at most the fractional $\preceq_{\text {Leb }}$-factor times $\max _{u \in V}\left|N_{\preceq \text { Leb }}[u]\right|$. Using an area bound, one can show that the closed $\preceq$ Leb-larger neighborhood of a disk in a set of disks of ply $\gamma$ has size at most $9 \cdot \gamma$ [24]. If the ply is 1 , the size is at most 7 . The theorem then follows from the fact that the fractional $\preceq_{\text {Leb }}$-factor for disk graphs is 6 .

The bound for planar graphs follows immediately from the above and the fact that planar graphs are disk graphs of ply 1 [21,24].

A PTAS for MDS on planar graphs is known [3], but we are not aware of any previous results on the integrality gap of the LP relaxation for this class of graphs.

By using Bar-Yehuda and Even's approximation algorithm for MSC instances of bounded element frequency [4], we can give a linear-time $(54 \cdot \gamma)$-approximation algorithm for Minimum Dominating Set on disk intersection graphs of ply $\gamma$.

We can improve on the $O(\gamma)$ ratio given above by using the shifting technique. One way is to approximate Minimum $\preceq$ Leb-Dominating Set.

Theorem 10. Minimum $\preceq_{L e b}$-Dominating Set on disk graphs of bounded ply, i.e. of ply $\gamma=\gamma(n)=o(\log n)$, has an FPTA $S^{\omega}$. Hence Minimum Dominating Set on disk graphs of bounded ply has a 6-FPTAA ${ }^{\omega}$.

The proof of Theorem 10 is omitted. Instead we use similar ideas to give a simpler algorithm for Minimum Dominating Set with better approximation ratio. The algorithm uses a new variant of the classic geometric shifting technique $[17,26]$. Assume the disks in a set $\mathcal{D}$ are scaled such that the smallest disk has radius $\frac{1}{2}$. Partition the disks into levels. A disk with radius $r$ has level $j\left(j \in \mathbb{Z}_{>0}\right)$ if $2^{j-1} \leq r<2^{j}$. The level of the largest disk is denoted by $l$. Define $\mathcal{D}_{=j}$ as the set of disks in $\mathcal{D}$ having level $j$. Similarly, $\mathcal{D}_{\geq j}$ denotes the set of disks having level at least $j$, and so on.

For each level $j$, define a grid by lines $y=h k 2^{j}$ and $x=v k 2^{j}(h, v \in \mathbb{Z})$ for some $k \geq 9$ (an odd multiple of 3 ), whose value we determine later. The grid partitions the plane into squares of size $k 2^{j} \times k 2^{j}$, called $j$-squares. A $j$-square is contained in precisely one $(j+1)$-square and each $(j+1)$-square contains exactly four $j$-squares. Let $\mathcal{D}^{S}$ denote the set of disks intersecting a $j$-square $S$ and $\mathcal{D}^{\mathrm{b}(S)}$ the set of disks intersecting the boundary of $S$. Similarly, $\mathcal{D}^{\mathrm{i}(S)}=\mathcal{D}^{S}-\mathcal{D}^{\mathrm{b}(S)}$ is the set of disks fully inside $S$. Combinations such as $\mathcal{D}_{=j}^{\mathrm{b}(S)}$ should be self-explanatory. The level of a square $S$ is denoted $j(S)$. Let $\mathcal{D}^{b}=\bigcup_{S} \mathcal{D}_{=j(S)}^{\mathrm{b}(S)}$ be the set of disks intersecting the boundary of a $j$-square at their level.

Theorem 11. Let $\mathcal{D}$ be a set of $n$ disks of ply $\gamma, k \geq 9$ an odd multiple of 3 , and OPT a minimum dominating set. Then in time $O\left(k^{2} n^{2} 3^{32 k \gamma / \pi} 2^{16 k \gamma / \pi} 4^{16(k+1) \gamma / \pi}\right)$, we can find a set $D S \subseteq \mathcal{D}$ dominating $\mathcal{D}-\mathcal{D}^{b}=\cup_{S} \mathcal{D}_{=j(S)}^{\mathrm{i}(S)}$ such that $|D S| \leq$ $\sum_{S}\left|O P T_{=j(S)}^{S}\right|$, where the union and the sum is over all squares $S$. 
The proof of this theorem is quite involved and is omitted due to space limitations.

The shifting technique is applied in the following novel way. For an integer $a(0 \leq$ $a \leq k-1)$, a line of level $j$ is active if it has the form $y=\left(h k+a 2^{l-j}\right) 2^{j}$ or $x=\left(v k+a 2^{l-j}\right) 2^{j}(h, v \in \mathbb{Z})$. The active lines partition the plane into $j$-squares as before, but are shifted w.r.t. $a$. However, we can still use the algorithm of Theorem 11.

Let $D S_{a}$ denote the set returned by the algorithm for the $j$-squares induced by $a$ and let $\mathcal{D}_{a}^{b}$ be the set $D^{b}$ for these $j$-squares $(0 \leq a \leq k-1)$. We join three such sets to ensure we dominate the entire graph. So let $D S_{i}^{3}=D S_{i} \cup D S_{i+k / 3} \cup D S_{i+2 k / 3}$ for each $i=0, \ldots, k / 3-1$. This is properly defined, as $k$ is a multiple of 3 . Denote the smallest $D S_{i}^{3}$ by $D S_{\min }^{3}$.

Theorem 12. There is a 3-FPTAA $A^{\omega}$ for Minimum Dominating Set on disk graphs of bounded ply, i.e. of ply $\gamma=\gamma(n)=o(\log n)$. If $\gamma=O(1)$, there is $a(3+\epsilon)$ approximation algorithm for any fixed $\epsilon>0$.

Proof. We first show $D S_{i}^{3}$ is a dominating set of $\mathcal{D}$, for any $i \in\{0, \ldots, k / 3-1\}$. A level $j$ disk is in $\mathcal{D}_{a}^{b}$ if and only if it intersects an active line of level $j$ for $a$. We know ([26], Lemma 9) that any disk intersects an active horizontal line for at most two (consecutive) values of $a$ and an active vertical line for at most two (consecutive) values of $a$. As $k \geq 9$ is an odd multiple of $3, k / 3>1$, and thus $i, i+k / 3, i+2 k / 3$ are non-consecutive integers (modulo $k$ ). Hence any disk is in at most two of the sets $\mathcal{D}_{i}^{b}, \mathcal{D}_{i+k / 3}^{b}, \mathcal{D}_{i+2 k / 3}^{b}$. Theorem 11 shows that $D S_{a}$ is a dominating set for $\mathcal{D}-\mathcal{D}_{a}^{b}$. Given the previous argument, $\left(\mathcal{D}-\mathcal{D}_{i}^{b}\right) \cup\left(\mathcal{D}-\mathcal{D}_{i+k / 3}^{b}\right) \cup\left(\mathcal{D}-\mathcal{D}_{i+2 k / 3}^{b}\right)=\mathcal{D}$. Then $D S_{i}^{3}$ is a dominating set of $\mathcal{D}$.

We now prove $\left|D S_{\min }^{3}\right| \leq\left(3+\frac{36}{k}\right)|O P T|$. A level $j$ disk is in $\mathcal{D}_{a}^{b}$ for at most 4 values of $a$ ([26], Lemma 9). Therefore $\sum_{a=0}^{k-1}\left|\mathcal{D}_{a}^{b}\right| \leq 4|\mathcal{D}|$ and $\sum_{a=0}^{k-1}\left|O P T \cap \mathcal{D}_{a}^{b}\right| \leq$ $4|O P T|$.

Also, for fixed $a$, any level $j$ disk intersects at most $4 j$-squares. Hence $\left|D S_{a}\right| \leq$ $\sum_{S}\left|O P T_{=j(S)}^{S}\right| \leq|O P T|+3\left|O P T \cap \mathcal{D}_{a}^{b}\right|$ and thus

$\frac{1}{3} k\left|D S_{\min }^{3}\right| \leq \sum_{i=0}^{k / 3-1}\left|D S_{i}^{3}\right| \leq \sum_{a=0}^{k-1}\left(|O P T|+3\left|O P T \cap \mathcal{D}_{a}^{b}\right|\right) \leq(k+12)|O P T|$.

Then $D S_{\min }^{3} \leq\left(3+\frac{36}{k}\right)|O P T|$. Choose $k$ as the smallest odd multiple of 3 greater than 9 and $\frac{36}{\epsilon}$ and apply Theorem 11 to get the $(3+\epsilon)$-approximation for constant ply and fixed $\epsilon$. The 3-FPTAA ${ }^{\omega}$ is obtained along similar lines using methods of [26].

Analogously, we can obtain 3-FPTAA ${ }^{\omega}$ 's for arbitrary fat objects of bounded ply. The algorithms of this section extend to $d$-dimensional fat objects for any constant $d$. We do not know if the shifting technique can be used to give a constant approximation (or even a PTAS) for MDS on disk graphs of arbitrary ply, because (1) there is no upper bound on the number of 'large' disks intersecting a $j$-square in the dominating set, and (2) we cannot track which $j$-square is 'responsible' for dominating a disk intersecting more than one $j$-square on its level. We avoided (1) by assuming bounded ply and (2) by considering $\preceq$ Leb -dominating sets (Thm. 10), or by disregarding the domination of disks intersecting a boundary on their level and combining three result sets (Thm. 12). 


\section{Hardness results}

The approximation schemes $[6,13,26]$ for Maximum Independent Set and Minimum Vertex Cover on disk intersection graphs extend easily to fat object intersection graphs. It is unlikely that an approximation algorithm for MDS would extend this way, as on intersection graphs of fat objects that are almost disks, MDS becomes hard to approximate. A convex subset $s$ of $\mathbb{R}^{2}$ is $\alpha$-fat for some $\alpha \geq 1$ if the ratio between the radii of the smallest disk circumscribing $s$ and the largest disk inscribed in $s$ is at most $\alpha$ [12].

Theorem 13. For any $\alpha>1$ and any $\epsilon>0, M D S$ on $\alpha$-fat object intersection graphs is not approximable within $(1-\epsilon) \ln n$, unless $N P \subset D T I M E\left(n^{O(\log \log n)}\right)$.

Proof. Reduce from Minimum Set Cover (MSC). For instance $x$ of MSC with universe $\mathbb{U}$ and collection $\mathcal{F}=\left\{S_{1}, \ldots, S_{m}\right\}$ of subsets of $\mathbb{U}$, construct instance $y$ of MDS on $\alpha$-fat object intersection graphs as in Fig. 2. Each $u \in \mathbb{U}$ corresponds to a 'small' disk $d_{u}$. Each $S_{j}$ corresponds to a disk $d_{j}$ with the top replaced by a polyhedral structure such that $d_{j}$ intersects $d_{u}$ if and only if $u \in S_{j}$. Packing the $d_{u}$ close together makes the fatness of the construction arbitrarily close to 1 . As any object dominated by a $d_{u}$ is also dominated by a $d_{j}$ for which $u \in S_{j}$, we have $\left|O P T_{x}\right|=\left|O P T_{y}\right|$. Constructing $y$ takes time polynomial in $|\mathbb{U}|$ and $m$. The theorem follows from Feige [15, 8].

An object has constant description complexity if it is a semialgebraic set defined by a constant number of polynomial (in)equalities of constant maximum degree [12]. The objects modeling the $S_{j}$ are the intersection of a disk with a polyhedron (each $d_{j}$ can be described by one quadratic inequality and $\left|S_{j}\right|+1$ linear inequalities) and might not have constant description complexity. So for constant description complexity objects, better approximation ratios than $\ln n$ could be attained. However, we can prove APXhardness by reducing from Minimum $k$-Set Cover, the variant of MSC where $\left|S_{j}\right| \leq k$ for any $S_{j} \in \mathcal{F}$. This problem is APX-hard for $k=3$ (follows e.g. from [1]). Using the same gadget as before, the objects of Theorem 13 have constant description complexity.

Theorem 14. For any $\alpha>1, M D S$ on $\alpha$-fat, constant description complexity object intersection graphs is APX-hard. Hence it has no PTAS (unless $P=N P$ ).

These results say something about intersection graphs of fat objects in general, and of fat almost disks in particular. But we can easily prove similar results for almost squares, almost bounded aspect ratio rectangles, almost triangles, etc. Basically, if we slightly relax the shape constraints for a given object, Minimum Dominating Set on the intersection graphs of such relaxed objects is hard to approximate.

The above reductions can also be used to prove the hardness of other problems, including Minimum Connected Dominating Set and Minimum Total Dominating Set. Furthermore, by replacing each disk $d_{u}$ in the reductions by a point, we obtain theorems equivalent to Theorem 13 and 14 for the $\ln n$-hardness of Geometric Set Cover on general $\alpha$-fat objects and $\alpha$-fat almost disks, almost squares, etc. and APX-hardness if these objects have constant description complexity.

Finally, we solve an open problem of Chlebík and Chlebíková [9] by proving that Minimum Dominating Set is APX-hard for intersection graphs of 2-dimensional boxes. The reduction can be extended to Minimum Connected/Total Dominating Set, to ellipse intersection graphs, and to Geometric Set Cover on rectangles and ellipses. 
Theorem 15. MDS on rectangle intersection graphs (MDSr) is APX-hard.

Proof. We give an L-reduction from the APX-hard [1] problem Minimum Vertex Cover on graphs $G=\left(\left\{v_{1}, \ldots, v_{n}\right\}, E\right)$ of degree three (MVC3) to MDS in a rectangle intersection graph $G^{\prime}$. Rectangles $R_{i}^{h}$ and $R_{i}^{v}$ represent vertex $v_{i}$, and are connected by three plates, the largest of which is the big plate $P_{i}$ (see Fig. 3). Edge $\left(v_{i}, v_{j}\right) \in E$ for $i<j$ corresponds to rectangle $S_{i, j}$ in the intersection of rectangles $R_{i}^{v}$ and $R_{j}^{h}$.

Let $C$ be a minimum vertex cover of $G$ and let $k=|C|$. Construct a set $D$ from $C$ by adding $R_{i}^{h}$ and $R_{i}^{v}$ to $D$ for each $v_{i} \in C$ and adding $P_{i}$ for each $v_{i} \notin C$. By the construction of $D$, all $R_{i}^{h}, R_{i}^{v}$, and all plates are dominated. As $C$ is a vertex cover, $D$ dominates each $S_{i, j}$. Since the graph has degree three, $|C| \geq n / 4$, and thus $|D| \leq$ $2|C|+(n-|C|)=n+k \leq 5 k$.

Let $D$ be a dominating set of $G^{\prime}$. We can assume that $D$ contains only rectangles of type $R_{i}^{h}, R_{i}^{v}$ and $P_{i}$. Construct a set $C$ from $D$ by adding $v_{i}$ to $D$ if $R_{i}^{h}$ or $R_{i}^{v}$ is in $D$. Because $D$ dominates all $S_{i, j}, C$ is a vertex cover. Let $R^{2}[D]$ be the set of rectangles for $v_{i}$ for which both $R_{i}^{h}$ and $R_{i}^{v}$ occur in $D, R^{1}[D]$ the set for $v_{i}$ for which only one of $R_{i}^{h}$ and $R_{i}^{v}$ occurs in $D$, and $P[D]$ the set of big plates in $D$. To dominate all small plates, $|P[D]|+\left|R^{2}[D]\right| / 2 \geq n$. Then $|D| \geq|P[D]|+\left|R^{1}[D]\right|+\left|R^{2}[D]\right| \geq$ $n+\left|R^{1}[D]\right|+\left|R^{2}[D]\right| / 2 \geq n+k$. Hence $O P T_{M D S r}\left(G^{\prime}\right)=n+k$. Suppose $|D|=$ $O P T_{M D S r}\left(G^{\prime}\right)+c$, for a certain $c \geq 0$. Then $|D|=n+k+c$ and thus $\left|R^{1}[D]\right|+$ $\left|R^{2}[D]\right| / 2+n \leq n+k+c$, implying $|C|-O P T_{M V C 3}(G) \leq c$.

\section{Conclusion}

The immediate open question is whether Minimum Dominating Set admits a constantfactor approximation algorithm or even a PTAS for disk graphs of arbitrary ply. The hardness results of Sect. 5 show that for objects whose boundaries can intersect an arbitrary number of times, MDS is very hard to approximate. On the contrary, if object boundaries intersect at most twice (i.e. the objects are pseudo-disks), the decomposition bound is linear and at least for cases such as $r$-regular polygons with constant $r$ or rectangles with bounded aspect-ratio, we get constant-factor approximation algorithms. An intriguing question is whether MDS on disk graphs is harder to approximate than for other intersection graph classes such as intersection graphs of squares, or whether the algorithmic ideas can be extended to disks or maybe even to arbitrary pseudo-disks (the decomposition bound starts to fail 'naturally' beyond pseudo-disks).

\section{References}

1. Alimonti, P., Kann, V., "Some APX-completeness results for cubic graphs", Theoret. Comput. Sci. 237 1-2 (April 2000), pp. 123-134.

2. Ambühl, C., Erlebach, T., Mihalák, M., Nunkesser, M., "Constant-Factor Approximation for Minimum-Weight (Connected) Dominating Sets in Unit Disk Graphs" in Proc. APPROXRANDOM 2006, LNCS 4110, Springer-Verlag, Berlin, 2006, pp. 3-14.

3. Baker, B.S., "Approximation Algorithms for NP-Complete Problems on Planar Graphs", J. ACM 411 (1994), pp. 153-180. 
4. Bar-Yehuda, R., Even, S., "A Linear-Time Approximation Algorithm for the Weighted Vertex Cover Problem", J. Algorithms 22 (June 1981), pp. 198-203.

5. Brönnimann, H., Goodrich, M.T., "Almost Optimal Set Covers in Finite VC-Dimension", Discrete Comput. Geometry 144 (1995), pp. 463-479.

6. Chan, T.M., "Polynomial-time Approximation Schemes for Packing and Piercing Fat Objects", J. Algorithms 462 (2003), pp. 178-189.

7. Chang, M.-S., "Efficient Algorithms for the Domination Problems on Interval and CircularArc Graphs", SIAM J. Comput. 276 (Dec. 1998), pp. 1671-1694.

8. Chlebík, M., Chlebíková, J., "Approximation Hardness of Dominating Set Problems" in Proc. ESA 2004, LNCS 3221, Springer-Verlag, Berlin, 2004, pp. 192-203.

9. Chlebík, M., Chlebíková, J., "The Complexity of Combinatorial Optimization Problems on d-Dimensional Boxes", SIAM J. Discrete Math. 211 (2007), pp. 158-169.

10. Clark, B.N., Colbourn, C.J., Johnson, D.S., "Unit Disk Graphs", Discrete Math. 86 1-3 (1990), pp. 165-177.

11. Clarkson, K.L., Varadarajan, K.R., "Improved Approximation Algorithms for Geometric Set Cover”, Discrete Comput. Geometry 371 (2007), pp. 43-58.

12. Efrat, A., Sharir, M., "The Complexity of the Union of Fat Objects in the Plane", Discrete Comput. Geometry 232 (2000), pp. 171-189.

13. Erlebach, T., Jansen, K., Seidel, E., "Polynomial-time Approximation Schemes for Geometric Intersection Graphs", SIAM J. Comput. 346 (2005), pp. 1302-1323.

14. Even, G., Rawitz, D., Sharar, S., "Hitting Sets when the VC-Dimension is Small", Inform. Process. Lett. 952 (2005), pp. 358-362.

15. Feige, U., "A Threshold of $\ln n$ for Approximating Set Cover", J. ACM 454 (1998), pp. 634-652.

16. Hochbaum, D.S., "Approximation Algorithms for the Set Covering and Vertex Cover Problems", SIAM J. Comput. 113 (August 1982), pp. 555-556.

17. Hochbaum, D.S., Maass, W., "Approximation Schemes for Covering and Packing Problems in Image Processing and VLSI", J. ACM 321 (1985), pp. 130-136.

18. Hunt III, D.B., Marathe, M.V., Radhakrishnan, V., Ravi, S.S., Rosenkrantz, D.J., Stearns, R.E., "NC-Approximation Schemes for NP- and PSPACE-Hard Problems for Geometric Graphs", J. Algorithms 262 (1998), pp. 238-274.

19. Kedem, K., Livne, R., Pach, J., Sharir, M., "On the Union of Jordan Regions and CollisionFree Translational Motion Amidst Polygonal Obstacles", Discrete Comput. Geometry 1 (1986), pp. 59-70.

20. Kim, S.-J., Kostochka, A., Nakprasit, K., "On the Chromatic Number of Intersection Graphs of Convex Sets in the Plane", Electr. J. Combinatorics 11 (2004), \#R52.

21. Koebe, P., "Kontaktprobleme der konformen Abbildung", Ber. Ver. Sächs. Ak. Wiss. Leipzig, Math.-Phys. Kl. 88 (1936), pp. 141-164.

22. Marathe, M.V., Breu, H., Hunt III, H.B., Ravi, S.S., Rosenkrantz, D.J., "Simple Heuristics for Unit Disk Graphs", Networks 25 (1995), pp. 59-68.

23. Marx, D., "Parameterized Complexity of Independence and Domination on Geometric Graphs" in Proc. IWPEC 2006, LNCS 4169, Springer-Verlag, Berlin, 2006, pp. 154-165.

24. Miller, G.L., Teng, S.-H., Thurston, W., Vavasis, S.A., "Separators for Sphere-Packings and Nearest Neighbor Graphs", J. ACM 441 (1997), pp. 1-29.

25. Nieberg, T., Hurink, J.L., "A PTAS for the Minimum Dominating Set Problem in Unit Disk Graphs" in Proc. WAOA 2005, LNCS 3879, Springer-Verlag, Berlin, 2006, pp. 296-306.

26. van Leeuwen, E.J., "Better Approximation Schemes for Disk Graphs" in Proc. SWAT 2006, LNCS 4059, Springer-Verlag, Berlin, 2006, pp. 316-327. 